Z. Klin. Chem. Klin. B3iochem.

11. Jg. 1973 , S. $537-542$

\title{
Die Bestimmung der $\gamma$-Glutamyltranspeptidase im Urin von nierengesunden und nierenkranken Personen mit einer automatischen kinetischen Methode
}

\author{
Von R. KLEY, G. BAhKe und H. HolZhürer \\ Aus dem Klinisch-Chemischen Zentrallaboratorium (Leiter: Prof. Dr. Dr. H. Greiling), der Abteilung Innere Medizin I \\ (Vorstand: Prof. Dr. S. Effert) und der Abteilung Innere Medizin II (Vorstand: Prof. Dr. R. Heinlz) \\ der Klinischen Anslalten der RWTH Aacben
}

(Eingegangen am 9. Juli/21. September 1973)

Es wird cine automatische kinetische Mcßmethode zur Aktivitätsbestimmung der Urin- $\gamma$-Glutamyltranspeptidase mit dem En\%ymautomaten 5010 (Firma Eppendorf) beschricben; die Zuverlässigkcitskriterien der Methode werden dargelegt. Bei 120 Nierengesunden wurden Normal werte des En\%yms im 24-h-Urin ermittelt, und dic Änderung der Ausscheidung bei 50 Nicrenkranken geprüft. Dic bei normalen Nierenfunktionen gemessenen hohen Aktivitäten fallen bei bestimmten Nierencrktankungen, unabhängig von der Genesc, signifikant auf niedrigere Werte ab. Sowohl bei Gesunden als auch bei Kranken war cine positive Korrelation zwischen der $\gamma$-GlutamyltranspeptidascAusschcidung pro Minute und der Kreatinin- b\%w. Harnstoff-Clearance nachwcisbar. Bei 60 Clearance-Untersuchungen $\%$ eigte es sich, dals dic $\gamma$-Glutamyltranspeptidase im Urin bei chronischen Nephropathien in $A$ bhängigkcit von dem funktionsfähigen Restparenchym vermindert ausgeschieden wird. Bei akuten Nephropathien konnte keine Abnahme der Enzymaktivität festgestellt werden.

\section{Determination of $\gamma$-glutamyl transpeptidase in the urine of normal persons and patients with kidney disease, using an automalic kinetic meellod}

An automatic kinctic method for the detcrmination of the activity of $\gamma$-glutamyl transpeptidase in urine by means of the en\%yme analy/cr 5010 (Eppendorf, Hamburg) is described and the reliability of the method shown. Normal values were obtained from 120 persons without renal diseases, and the variation of these values was studied in the urines of 50 persons with renal diseases. The normally high activitics are significantly decreased in patients with renal diseases, independent of the origin of the discasc. $\Lambda$ positive correlation between the excretion rate of $\gamma$-glutamyl transpeptidase and creatinine or urea clearance respectively was shown both in the presence and in the absence of renal disease. With 60 clearance studics it could be shown that the $\gamma$-glutamyl transpeptidase excretion in urine from patients with chronic nephropathias was decreased in proportion to the remaining active parenchyma. With acute nephropathias, however, no decrease of the cnzyme activity was observed.

Die $\boldsymbol{y}$-Glutamyltranspeptidase, ein Enzym des aktiven Aminosäuretransportes, läßt sich in verschiedenen menschlichen Geweben und im Scrum nachweisen (1-4). Die höchsten Aktivitäten finden sich in der Niere und im Urin (5-8). Die im menschlichen Harn nachweisbare $\gamma$-Glutamyltranspeptidase ist eine lösliche Form des in den Bürstensäumen der proximalen Nicrentubuli membranös gebundenen Enzyms (12). Seit den Arbeiten von OrLowskr, SzewCZuK und Szasz sind die methodischen Voraussetzungen für die Messung der $\gamma$-Glutamyltranspeptidase im. menschlichen Urin gegeben. Lévy, Dubach und Thiele $(7,8)$ fanden bei chronischen Nierenerkrankungen eine deutliche Verminderung der $\gamma$-Glutamyltranspeptidase im Urin. Im folgenden berichten wir über Untersuchungen der Urin- $\gamma$-Glutamyltranspeptidase bei nierengesunden und nierenkranken Personen mit Hilfe einer automatischen kinetischen Meßmethode.

\section{Untersuchungsgut}

Zur Ermittlung von Normalwcrten wurde dic Aktivität der $\gamma$ Glutamyltranspeptidase in 24-h-Urin von 120 nierengesunden Personen bestimmt (poliklinische Patienten und Laboratoriumspersonal), 58 davon waren Frauen. Dic Auswahl erfolgte aufgrund klinischer und labormedizinischer Untersuchungen.
Bei 60 überwiegend Langzeit-Clearance-Untersuchungen, 45 Nephropathien und 15 Nierengesunden, haben wir die Aktivität des Enzyms gemesscn. Dic Änderung der $y$-Glutamyltranspeptidasc-A usscheidung im 24-h-Urin prüften wir bei 50 Nephropathien unterschiedlicher Genese (25 ₹, 25 ろ).

\section{Methodik}

Es wurden 24-h-Urinc ohne Konscrvicrungszusätze gesammelt. Ein aliquoter Teil des durchmischten Urins wurde $10 \mathrm{~min}$ lang bei $4000 \mathrm{~g}$ abzentrifugicrt und der klare Ubcrstand weiterverarbeitct.

Um vergleichbare und reproduzicrbare Ergcbnissc zu crhalten, haben wir die Messung der $\gamma$-Glutamyltranspeptidase-Aktivität im Urin mit ciner automatisierten kinctischen Meßmethode im Enzymautomaten 5010 (Firma Eppendorf, Gerätebau, Hamburg) durchgeführt. Bei Anwendung von $\gamma$-Glutamyl-p-nitranilid als Substrat und Glycylglycin als Akzeptor sind nach den Untersuchungen von Srsasz (6) die optimalen Reaktionsbedingungen für dic $\gamma$-Glutamyltranspeptidase im Urin von denen der $\gamma$ Glutamyltranspeptidasc im Scrum nur unwesentlich verschicden. Testansatz und Meßbedingungen wurden deshalb für dic Messung im Urin nicht verändert. In der Tabelle 1 sind dic wichtigsten Angaben über dic automatisierte Mc(3methode zusammengefaßt. Im Urin muß nach Szaš (6) mit dem Vorkommen hitzestabiler und dialysicrbarer Inhibitoren gerechnet werden. Um den Einfluß dieser möglichen Inhibitoren auszuschlicßen, haben wir dic Urine mit Hilfe der "Hollow-Piber-Technique" dialysicrt (9).

Ein Bio-Fibcr-50-Bcakcr (Firma Bio-Rad-Laboratorics, USA) wurde mit $70 \mathrm{ml}$ cines 24 -h-Urins gefüllt. Dic Dialyse erfolgte 
Tab. 1

Methodische Angaben zur automatischen, kinetischen Bestimmungsmethode der Urin- $\gamma$-Glutamyltranspeptidase mit dem Enzymautomaten 5010 (Fa. Eppendori, Hamburg)

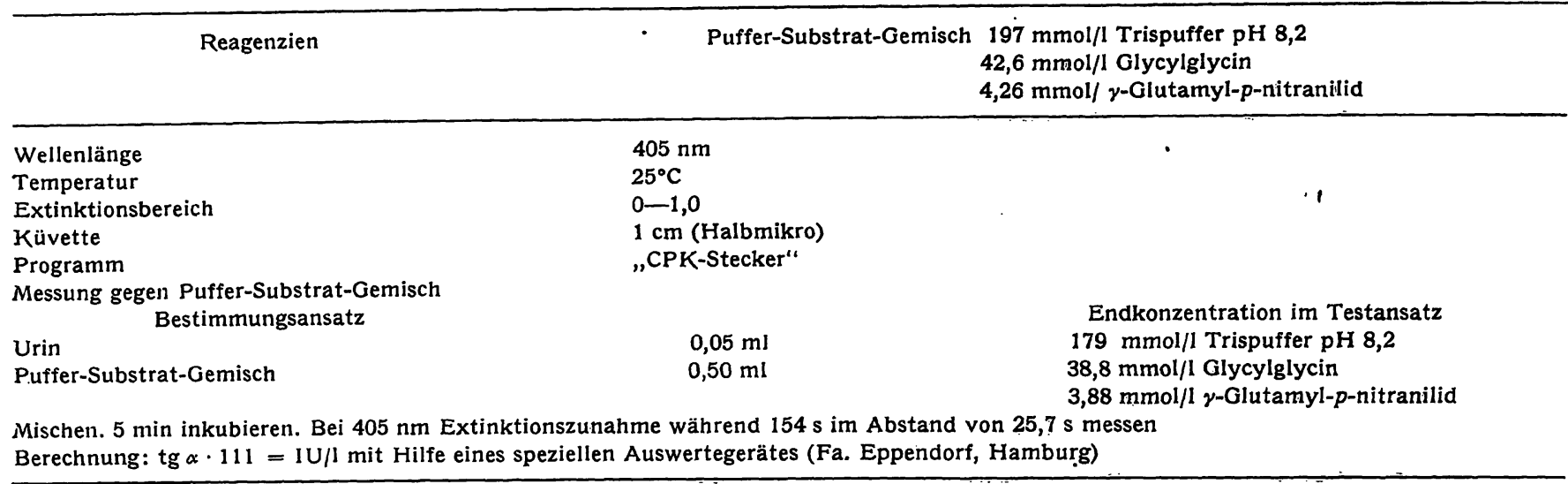

unter ständigem Rühren bei einer Temperatur von $20^{\circ} \mathrm{C}$ gegen demineralisiertes Wasser, das mit einer Geschwindigkeit von etwa $100 \mathrm{ml} / \mathrm{min}$ durch das Cellulose-Fiber-Bündel des Beakers geleitet wurde. Durch laufende Messungen der elektrischen Leitfähigkeit der Urinproben konnten die Dialysezeiten, die zwischen 100 und 300 min schwankten, für jede Probe individuell festgelegt werden. Nach Beendigung der Dialyse wurde der Beaker sorgfältig entleert, das Urinvolumen gemessen und die Aktivität des Enzyms bestimmt: Mit diesem Verfahren war es möglich, Substanzen mit einem Molekulargewicht zwischen 1000 und 10000 aus der Harnprobe zu entfernen (9).

Zum Vergleich der $\gamma$-Glutamyltranspeptidase-Aktivitäten in Nativharn, dialysierten und chromatographierten Urinen wurden in Parallelversuchen die Proben einer Gelchromatographie unterworfen, durch die auch höher molekulare Inhibitoren oder Aktivatoren vom Enzym abgetrennt werden können (10). Um optimale Chromatographiebedingungen zu erhalten (11), haben



Schema der Aufarbeitungsschritte für die Gelchromatographie der Urin- $\gamma$-Glutamyltranspeptidase wir wegen des hohen Molekulargewichtes der $\gamma$-Glutamyltranspeptidase (12) als Säulenmaterial Biogel P 100 (Bio-Rad-Laboratories, USA) gewählt, dessen Trennbereich für globuläre Proteine und Peptide bei einem Molekulargewicht zwischen $1 \cdot 10^{4}$ und $1 \cdot 10^{5}$ liegt. In der Abbildung 1 sind schematisch die wichtigsten Aufarbeitungsschritte der Urine für die Säulenchromatographie aufgezeichnet. Ein typisches Elutionsdiagramm mit Angaben über die Chromatographiebedingungen zeigt. die Abbildung 2.

Die $\gamma$-Glutamyltranspeptidase-Aktivitäten wurden unter Berücksichtigung des ausgeschiedenen Urinvolumens in IU/24 h angegeben.

Die Harnstoff- und Kreatinin-Werte wurden mit Hilfe eines SMA 12/60 Autoanalyzers (Firma Technicon Corp.) bestimmt.

Wegen der unterschiedlichen Verteilungsmerkmale der einzelnen überprüften Parameter haben wir für die Korrelationsanalysen den verteilungsfreien Rang-Korrelations-Koeffizienten ( $\left.\boldsymbol{t}_{\mathbf{s}}\right)$ nach Spearman und für den Mittelwertvergleich von Stichproben den U-Test nach Wilcoxon, MANN und WhItNEY (13) angewendet.



Abb. 2

Chromatogramm des 24-h-Urins eines nierengesuriden Probanden auf Biogel P $100(39 \times 3 \mathrm{~cm})$. Elutionsmittel: $0,5 \mathrm{~mol} / \mathrm{l} \mathrm{NaCl}$. Elutionsgeschwindigkeit: $15 \mathrm{ml} / \mathrm{h}$. Temperatur: $+4^{\circ} \mathrm{C}$. Die Bestimmung der Proteinkonzentration erfolgte mit dem Fol.iN-Phenol-Reagenz nach O $\gamma$-Glutamyltranspeptidase; $\triangle$ Protein 
Tab. 2

Richtigkeitsprüfung der Urin- $y$-Olutamyltranspeptidase-Bestimmungsmethode auf dem Enzymautomaten 5010 (Fa. Eppendorf, Hamburg)

\begin{tabular}{|c|c|c|c|c|}
\hline & $\begin{array}{c}\text { Sollwert } \mathbf{X}_{\mathrm{B}} \\
(\mathrm{U} / \mathrm{l})\end{array}$ & Analysenzah] & $\begin{array}{c}\text { Gefundener Wert } \mathbf{X}_{\mathbf{L}} \\
(\mathrm{U} / \mathrm{l})\end{array}$ & $\frac{X_{s}-X_{1}}{X_{s}} \cdot 100(\%)$ \\
\hline$\cdot$ & $\begin{array}{l}110 \\
55 \\
22,5 \\
6,3 \\
2,0\end{array}$ & $\begin{array}{l}20 \\
20 \\
10 \\
20 \\
20\end{array}$ & $\begin{array}{r}110 \\
57,3 \\
22,2 \\
5,3 \\
2,0\end{array}$ & $\begin{array}{l} \pm 0 \\
-4,1 \\
+1,3 \\
+15,8 \\
\pm 0\end{array}$ \\
\hline
\end{tabular}

Präzision der Urin- $\gamma$-Glutamyltranspeptidase-Bestimmungsmethode mit dem Enzymautomaten 5010 (Fa. Eppendorf, Hamburg)

\begin{tabular}{|c|c|c|c|c|c|}
\hline Urin & Bestimmungsmodus & Analysenzahl & $\begin{array}{l}\text { Mittelwert } \\
(U / 1)\end{array}$ & $\begin{array}{c}2 s \\
(U / 1)\end{array}$ & $\begin{array}{c}\text { Variationskoeffizient } \\
(\%)\end{array}$ \\
\hline \multirow[t]{2}{*}{1} & in Serie & 30 & 50,0 & $\pm 3,0$ & 3,0 \\
\hline & von Tag zu Tag & 40 & 49,0 & $\pm 4,0$ & 4,0 \\
\hline \multirow[t]{2}{*}{ II } & in Serie & 30 & 69,0 & $\pm 4,0$ & 2,8 \\
\hline & von Tag zu Tag & 40 & 63,0 & $\pm 5,0$ & 3,9 \\
\hline \multirow[t]{2}{*}{ III } & in Serie & 60 & 45,5 & $\pm 2,0$ & 2,1 \\
\hline & von Tag zu Tag & 40 & 46,5 & $\pm 3,0$ & 3,2 \\
\hline
\end{tabular}

\section{Spezifität}

Entsprechend den Untersuchungen von ORLowskI (14) und Szasz (6) ist die Methode spezifisch für die $\gamma$-Glutamyltranspeptidase im Urin.

\section{Richtigkeit}

Der Vergleich zwischen unserer automatisierten und der manuellen Urin- $\gamma$-Glutamyltranspeptidase-Bestimmung nach SzAsz (6) ergibt eine gute Ubereinstimmung $\left(r_{s}=0,9\right)$. In der Tabelle 2 ist das Ergebnis der Richtigkeitsprüfung zusammengefaßt.

\section{Linearität}

Durch Verdünnungsreihen von chromatographisch gereinigten $\gamma$-Glutamyltranspeptidase-Fraktionen, dialysiertem und nicht dialysiertem Harn wurde die Linearität der Methode überprüft. Ein Vergleich der Ist- und Sollwerte zeigt eine gute Linearität im Bereich zwischen 2,0 und $110 \mathrm{U} / 1$.

\section{Präzision}

Sowohl für die Präzision in Serie wie auch von Tag zu Tag lag der Variationskoeffizient zwischen 2,1 und 4\% (s. Tab. 3).

\section{Driftkontrolle, Verscbleppungsfebler und Substrateigenbydrol'se}

$\mathrm{Zu}$ Anfang, in der Mitte und am Ende einer Analysenreihe wurden Segmente mit Kontrollurinen eingefügt, bei Einbaltung der Betriebsbedingungen des Enzymautomaten konnte keine Drift festgestellt werden. Ähnlich wie bei der Driftkontrolle wurden solche Segmente eingefügt, in denen sich Urinproben mit hoher Enzymaktivität und Wasserproben abwechselten. Eine Verschleppung wax nicht meßbar. Bei intakter Substratlösung fanden wir in Ubereinstimmung mit SzAsz eine Eigenhydrolyse unter $1,0 \mathrm{U} / 1$.

Die Probenfrequenz betrug 120 Analysen/h.

\section{Ergebnisse}

Beim Vergleich der $\gamma$-Glutamyltranspeptidase-Aktivitäten vor und nach Dialyse waren im Durchschnitt keine signifikanten Erhöhungen der Enzymaktivitäten nach der Dialyse zu registrieren.

Lediglich bei 4 Urinproben konnte eine Hemmung des Enzyms zwischen 10 und 15\% festgestellt werden.

Auch nach Chromatographie auf Biogel $\mathrm{P} 100$ zeigte die $\gamma$-Glutamyltranspeptidase keine. Steigerung ihrer Ak-

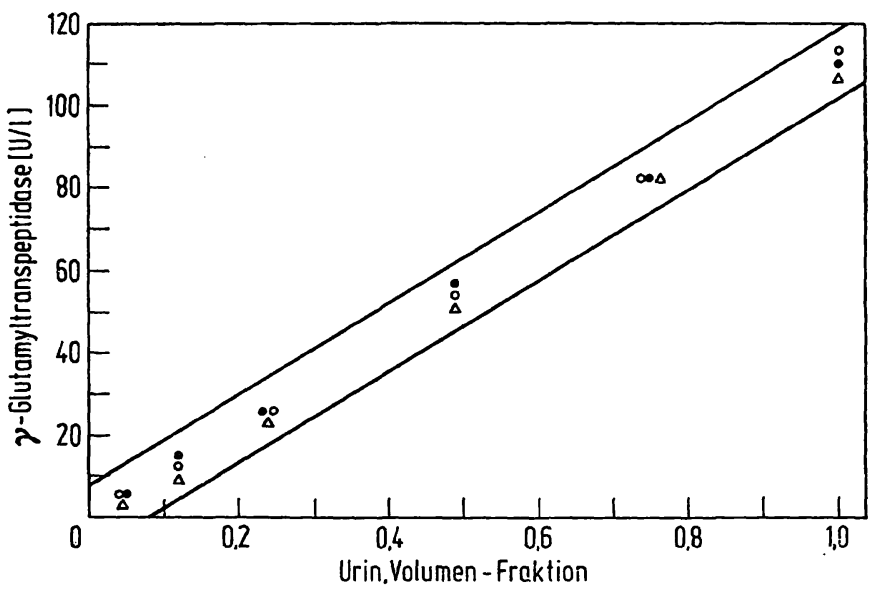

Abb. 3

Vergleich der $\gamma$-Glutamyltranspeptidase-Aktivitäten von Nativharn, dialysiertem und chromatographiertem Harn aus einer Urinprobe. Die unverdünnte Konzentration der Urine auf der Abszisse ist 1,0

- Nativharn; O chromatographierter Harn; $\Delta$ dialysierter Harn

tivität. Die Abbildung 3 zeigt, daß bei dem von uns gewählten Testansatz zwischen Nativharn und chromatographiertem Urin nur eine geringe Abweichung besteht. Eine Hemmung der chromatographisch abgetrennten $\boldsymbol{\gamma}$-Glutamyltranspeptidase durch Inkubation mit Nativharn konnte nicht nachgewiesen werden.

\section{$\gamma$-Glutamyltranspeptidase-Aktivität im Harn nierengesunder Personen}

Das Alter des untersuchten nierengesunden Kollektivs lag $z$ wischen 16 und 75 Jahren. Eine besondere Altersverteilung konnte nicht festgestellt werden. In der Tabelle 4 sind die Normalwerte der $\gamma$-Glutamyltranspeptidase im 24-h-Urin, wie sie von verschiedenen Untersuchern ermittelt wurden, vergleichend aufgeführt. •

Bei Gesunden bestand eine signifikante Korrelation $z$ wischen der $\gamma$-Glutamyltranspeptidase-Ausscheidung und dem Harnvolumen; keine Korrelation dagegen sahen wir zwischen täglich ausgeschiedener Kreatinin- 
Tab. 4

Normalwerte der $\gamma$-Glutamyltranspeptidase im 24-h-Urin, ermittelt von verschiedenen Untersuchern an nierengesunden Personen

$\mathrm{N}=$ Anzahl der Probanden, $\overline{\mathrm{x}}=$ Mittelwert, $\mathrm{s}=$ Standardabweichung von $\overline{\mathrm{x}}, 95 \% \mathrm{VB}=$ unsymmetrischer $95 \%$ Vertrauensbereich

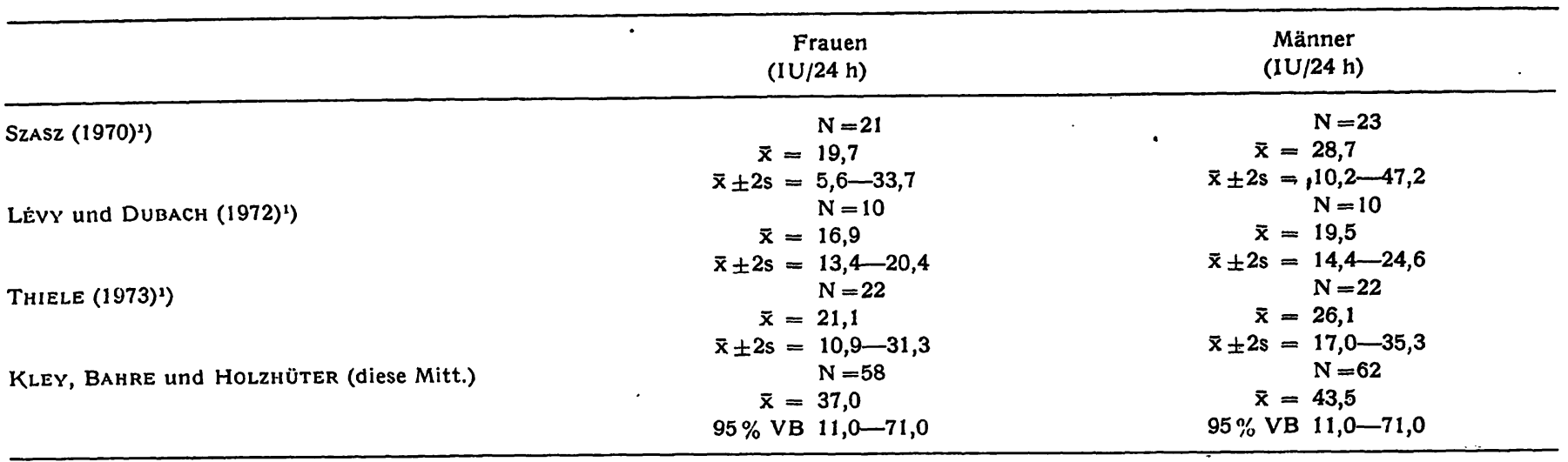

2) Die in IU/8 h angegebenen Originaldaten wurden mit dem von SzASz (6) empfohlenen Faktor 2,64 auf $24 \mathrm{~h}$ umgerechnet.

Tab. 5

Übersicht über die Korrelationen zwischen Urin- $\gamma$-Glutamyltranspeptidase und anderen von der Nierenfunktion abhängigen Parametern. Die statistische Berechnung erfolgte mit den im Text angegebenen Methoden

$r_{s}=$ SPEARMAN-Korrelationskoeffizient $x_{1}=$ Signifikanzniveau von $r_{s}$

\begin{tabular}{llcc}
\hline Korrelation zu & Nieren- & \multicolumn{1}{c}{ r $_{\text {s }}$} & $\alpha$ \\
\hline Harnvolumen & gesund & 0,697 & $<0,001$ \\
& krank & $-0,334$ & 0,05 \\
Urin-Kreatinin & gesund & 0,291 & 0,100 \\
& krank & 0,306 & 0,05 \\
Serum-Kreatinin & gesund & $-0,321$ & 0,05 \\
& krank & $-0,580$ & $<0,001$ \\
Serum-Harnstoff & gesund & $-0,306$ & 0,05 \\
\multirow{3}{*}{ Kreatinin-Clearance } & krank & $-0,576$ & $<0,001$ \\
& gesund & 0,521 & 0,005 \\
Harnstoff-Clearance & krank & 0,785 & $<0,001$ \\
& gesund & 0,501 & 0,005 \\
& krank & 0,720 & $<0,001$ \\
\hline
\end{tabular}

menge und dem Enzym. Aus diesem Grunde haben wir als Bezugsbasis für die Aktivitätsangaben der Urin$\gamma$-Glutamyltranspeptidase das 24-h-Volumen gewählt. Zwischen Clearance-Größen und der $\gamma$-Glutamyltranspeptidase bestehen signifikante positive Korrelationen (siehe Tab. 5).

\section{$\gamma$-Glutamyltranspeptidase-Aktivität im Urin nierenkranker} Personen

Das von uns untersuchte Krankengut ließ sich in folgende Nephropathien einteilen:

1. vorwiegend glomeruläre Erkrankungen mit Niereninsuffizienz (13 Fälle)

2. vorwiegend interstitielle Erkrankungen ohne Niereninsuffizienz (10 Fälle)

3. vorwiegend interstitielle Erkrankungen mit Niereninsuffizienz (13 Fälle)

4. andere Erkrankungen mit Niereninsuffizienz (14 Fälle).

Gegenüber der gesunden Vergleichsgruppe ergaben sich für diese Nierenerkrankungen, unabhängig vom Alter und Geschlecht der Patienten, statistisch signifikant niedrigere Enzymaktivitäten im 24-h-Urin. Im U-Test nach Wircoxon et al. (13) waren die Mittelwerte der obigen Krankheitsgruppen gegenüber dem Normalkollektiv mit einer 95 prozentigen Sicherheit erniedrigt $(\hat{z}>U=1,96, \quad \alpha=0,05)$. Die Enzymaktivitäten schwankten zwischen 1000 und $12000 \mathrm{IU} / 24 \mathrm{~h}$, unabhängig von der Genese der Erkrankung.

Zwischen dem Harnvolumen und der $\gamma$-Glutamyltranspeptidase-Ausscheidung konnten wir keine Kor-

Tab. 6

Sieben Beispiele von Kreatinin- und Harnstoffuntersuchungen bei gleichzeitiger Messung der $\gamma$-Glutamyltranspeptidase-Aktivität im Urin

\begin{tabular}{|c|c|c|c|c|c|c|}
\hline Diagnose & $\begin{array}{c}\text { Clearancedauer } \\
\text { (h) }\end{array}$ & $\begin{array}{l}\text { Serum-Kreatinin } \\
(\mu \mathrm{mol} / \mathrm{l})\end{array}$ & $\begin{array}{l}\text { Kreatinin- } \\
\text { Clearance } \\
(\mathrm{ml} / \mathrm{s})\end{array}$ & $\begin{array}{l}\text { Serum-Harnstoff } \\
(\mathrm{mmol} / \mathrm{l})\end{array}$ & $\begin{array}{l}\text { Harnstoff- } \\
\text { Clearance } \\
(\mathrm{ml} / \mathrm{s})\end{array}$ & $\begin{array}{l}\gamma \text {-Glutamyl- } \\
\text { transpeptidase } \\
\text { (IU/s) }\end{array}$ \\
\hline \multirow[t]{3}{*}{ Akute Pyelonephritis } & 2 & 97,4 & 4,9 & 9,3 & 2,6 & 0,85 \\
\hline & 2 & & 4,5 & & 1,5 & 0,66 \\
\hline & 2 & & 3,1 & & 1,2 & 0,66 \\
\hline Akute Glomerulonephritis & 2 & 88,5 & 4,3 & 9,1 & 1,7 & 1,65 \\
\hline \multicolumn{7}{|l|}{ Niereninsuffizienz b. chron. } \\
\hline \multicolumn{7}{|l|}{ Niereninsuffizienz b. chron. } \\
\hline Pyelonephritis & 2 & 354,0 & 0,6 & 15,0 & 0,5 & 0,63 \\
\hline Analgetica-Abusus & 2 & 88,5 & 6,4 & 8,3 & 2,7 & 0,67 \\
\hline Cystenniere & 24 & 106,2 & 1,8 & 10,3 & 0,6 & 0,27 \\
\hline Nierentumor li. & 12 & 88,5 & 7,5 & 6,6 & 3,7 & 1,27 \\
\hline
\end{tabular}


relation feststellen, ebenso nicht zwischen der täglich ausgeschiedenen Kreatininmenge und dem Enzym. Zwischen Serumkreatinin- bzw. Serumharnstoff-Spiegel und der $\gamma$-Glutamyltranspeptidase-Aktivität im Urin bestand eine signifikante Korrelation: Bei Erhöhung dieser beiden Parameter im Serum sank die $\gamma$-Glutamyltranspeptidase ab. Bei Gesunden und Nierenkranken war außerdem eine signifikante positive Korrelation $z$ wischen $\gamma$-Glutamyltranspeptidase-Ausscheidung und der Kreatinin- bzw. Harnstoff-Clearance nachweisbar (s. Tab. 5). In der Tabelle 6 sind typische Beispiele für Clearance-Untersuchungen bei verschiedenen Nephropathien aufgeführt. Die $\gamma$-Glutamyltranspeptidase-Ausscheidung schwankt hier, insbesondere bei KurzzeitUntersuchungen, $z$ wischen Normalwerten und pathologisch erniedrigten Aktivitäten. Bei Nierengesunden fanden wir als unteren Grenzwert der Enzymaktivität $0,27 \mathrm{U} / \mathrm{s}$.

\section{Diskussion}

Wie bei allen anderen Urinenzymen sind auch bei der Aktivitätsmessung der $\gamma$-Glutamyltranspeptidase eine Reihe von Schwierigkeiten zu beachten: neben den unterschiedlichen $\mathrm{pH}$-Werten und den verschiedenen Elektrolytkonzentrationen, die zu einer Veränderung der Enzymaktivität führen können, fehlt eine exakt definierte Bezugsgröße; je nach Flüssigkeitsaufnahme und Transpiration sind Urinvolumina zu erwarten, die in 'entsprechenden Grenzen variieren. Hinzu kommen die Sammelfehler, die bei der Wahl kurzer Sammelperioden besonders stark ins Gewicht fallen. Ähnlich wie Lévy, Thiele und Szasz fanden wir bei Nierengesunden eine deutliche Abhängigkeit der $\gamma$-Glutamyltranspeptidase-Aktivität im 24-h-Urin vom jeweiligen Harnvolumen.

Ein besonderer Unsicherheitsfaktor bei der Messung von Urinenzymen besteht in der möglichen Anwesenheit von Inhibitoren oder Aktivatoren, wodurch eine aufwendige Vorbereitung der Harnproben erforderlich und eine routinemäßige Anwendung erschwert wird. Spezifische Inhibitoren sind z. B. für die Lactatdehydrogenase im Urin nachgewiesen und von SCHOENENBERGER und WACKER isoliert worden (16). Beide Untersucher stellten fest, daB Inhibitoren im Urin erst bei einem Probevolumen von mehr als einem Fünftel des Gesamtvolumens im Testansatz die Messungen stören können. Für die $\gamma$-Glutamyltranspeptidase wurden von SZASZ (6) ein oder mehrere niedermolekulare, hitzestabile Inhibitoren postuliert, deren Isolierung und nähere Charakterisierung jedoch noch aussteht (17). Entsprechend der Empfehlung von SCHOENENBERGER et al. haben wir das Urinvolumen im Bestimmungsansatz klein gehalten (s. Tab. 1). SzAsz (6) beobachtete mit dem gleichen Testansatz bei $40 \%$ der Messungen eine Hemmung des Enzyms, während Threle (8) eine durchschnittliche Zunahme der Enzymaktivitäten nach Dialyse der Proben von 5\% fand. Die Anzahl der von uns beobachteten Hemmungen der Urin- $\gamma$-Glutamyl- transpeptidase lag unter $2 \%$. Statistisch fanden wir im Durchschnitt in den von uns untersuchten Harnproben keine signifikante Zunahme der Enzymaktivität nach Dialyse und Gelchromatographie. Prinzipiell kann man aufgrund dieser Ergebnisse Nativharn nach Abzentrifugieren direkt zur Bestimmung einsetzen, was für die Routinediagnostik eine erhebliche Reduzierung des Arbeitsaufwandes bedeutet. Darüberhinaus ist es mit der von uns angewandten automatisierten kinetischen Meßmethode möglich, die Präzision der Bestimmung zu verbessern. Allerdings muß damit gerechnet werden, $\mathrm{da} B$ trotzdem einige Ergebnisse durch eventuell vorhandene Inhibitoren usw. verfälscht werden.

Szasz und ThIELE nehmen eine weitgehende Normalverteilung der $\boldsymbol{\gamma}$-Glutamyltranspeptidase im Urin an. Die statistische Auswertung unseres größeren Kollektivs ergab keine Normalverteilung. Im Gegensatz zum Serumenzym, von dem eine lognormale Verteilungsform her bekannt ist $(18,19)$, konnten wir für die Urin- $\gamma$ Glutamyltranspeptidase diese nicht sicher nachweisen. Deshalb haben wir den unsymmetrischen $95 \%$ Vertrauensbereich der Enzymausscheidung bei Nierengesunden als Normwertgrenzen angegeben (s. Tab. 4). Trotz der unterschiedlichen Mittelwerte ließ sich statistisch kein signifikanter Unterschied $z$ wischen der $\gamma$-Glutamyltranspeptidase-Aktivität im 24-h-Urin bei gesunden Männern und Frauen feststellen. Hierzu muß allerdings gesagt werden, daß im U-Test nach WILcoxon die Prüfgröße $\hat{z}=1,945$ nur knapp unter dem Schwellenwert $U=1,96(\alpha=0,05)$ lag und deshalb die Hypothese der Gleichheit der Mittelwerte nicht verworfen werden konnte. Die aus der Tabelle $4 \mathrm{zu}$ ersehenden Abweichungen der Normalwerte einzelner Untersucher lassen sich in erster Linie durch die kleine Probandenzahl und durch die Zugrundelegung einer Normalverteilung bei der Berechnung erklären.

Ebenso wie THIELE konnten wir bei den ClearanceUntersuchungen von chronischen Nierenerkrankungen eine signifikante Abhängigkeit der Enzymaktivität von der Kreatinin- und Harnstoff-Clearance zeigen. Diese Abhängigkeit läßt sich dadurch erklären, daß bei chronischen Nephropathien das funktionsfähige Restparenchym mit der Menge des Glomerulumfiltrates korreliert. Bei den Clearance-Untersuchungen bei relativ wenigen akuten Nierenerkrankungen waren die $\gamma$-Glutamyltranspeptidase-Aktivitäten nicht vermindert, aber auch nicht vermehrt, obwohl die Harnstoff- und Kreatinin-Clearance bereits pathologisch ausfielen (s. Tab. 6). Es muß jedoch betont werden, daß einerseits die Zahl der Clearance-Untersuchungen bei akuten renalen Erkrankungen niedrig lag, wodurch statistische Aussagen schlecht möglich sind und andererseits die Dauer der Clearance-Untersuchungen kurz waren, so daß unterschiedliche Diuresen, eventuelle tageszeitliche Schwankungen und Sammelfehler besonders stark ins Gewicht fallen. Wurde die Sammelperiode auf $24 \mathrm{~h}$ verlängert, waren sowohl bei chronischen wie auch bei akuten Nierenerkrankungen die Enzymaktivitäten deutlich vermindert. 
LÉvy (7) und THIELE (8) beobachteten bei einigen Nephropathien hohe $\gamma$-Glutamyltranspeptidase-Aktivitäten im Urin. Wir konnten an einem größeren Kollektiv histologisch gesicherter chronischer : Glomerulonephritiden ohne Niereninsuffizienz ebenfalls eine deutliche Enzymurie beobachten. Bei Verlaufskontrollen zeigten viele Patienten dieser Krankheitsgruppe $z$ wischenzeitig auch normale $\gamma$-GlutamyltranspeptidaseWerte im 24-h-Urin. Über die klinische Bedeutung dieser Befunde ist an anderer Stelle berichtet worden (20).

Aufgrund der zitierten Untersuchungen und unserer eigenen Ergebnisse besteht ein kausaler Zusammenhang zwischen' Nierenfunktion und Urin- $\gamma$-Glutamyltranspeptidase-Aktivität, der sich aus der physiologischen Bedeutung des Enzyms für den aktiven Transport der
Aminosäuren in der Niere ableiten läßt (1). Störungen dieses Transportsystems durch pathologische Veränderungen im Nierenparenchym haben eine entsprechende Einwirkung auf die $\gamma$-Glutamyltranspeptidase-Ausscheidung im Harn. Weitere Untersuchungen müssen zeigen; ob sich die $\gamma$-Glutamyltranspeptidase im Urin zur Verlaufskontrolle bei Nierenerkrankungen eignet; differentialdiagnostische Schlüsse auf die Art der Nierenerkrankung sind anhand einzelner Enzymwerte sicherlich nicht möglich.

\section{Danksagung}

Herrn Dr. H. Stuhlsatz und Herrn Priv.-Doz. Dr. E. Wenzel seien für die wertvollen Diskussionen, und Herrn Dr. BuschSIEWEKE (Abteilung für Med. Statistik und Dokumentation der RWTH Aachen) für die statistischen Beratungen und für die Berechnungen gedankt.

\section{Literatur}

1. Orlowskr, M. \& Merster, A. (1970), Proc. Nat. Acad. Sci. USA 67, 1248-1255. - 2. SzewCZuK, A. \& OrLowskI, M. (1960), Clin. Chim. Acta 5, 680-688. - 3. OrLowskI, M. \& Szewyczuk, A. (1961), Clin. Chim. Acta 6, 430-432. 4. Orlowski, M. \& Szewczuk, A. (1961), Acta Biochim. Pol. 8, 189-200. - 5. OrLowskx, M. \& SzewCzUK, A. (1962), Clin. Chim. Acta 7, 755-760. - 6. Szasz, G. (1970), diese Z. 8, 1-8. 7. LÉVY, A. \& Dubach, U. C. (1972), Klin. Wochenschr. 50, 438-441. - 8. Thiele, K. G. (1973), Klin. Wochenschr. 51, 339-345. - 9. Bio-Rad-Laboratories (1972), "The New Hollow Fiber Technique", Technical Bulletin Nr. 1004, RichmondCalifornia (USA). - 10. DetermanN, H. (1967), Gelchromatographie S. 93, Verlag Springer, Berlin-Heidelberg-New York. 11. FISCHER (1969), An introduction to gel chromatography, S. 189 u. 223, North Holland Publishing Comp. Amsterdam-
London. - 12. Szewczuk, A. (1966), Clin. Chim. Acta 14, 608-614. - 13. SACHS, L. (1972), Statistische Auswertungsmethoden, 3. Aufl. S. 230-312, Verlag Springer, Berlin-Heidelberg-New York. - 14. OrLowskr, M. \& MeIster, A. (1963), Biochim. Biophys. Acta 73, 679-681. - 15. LowRX, O. H., Rosebrough, N. J., Farr, A. L. \& Randall, R. J. (1951), J. Biol. Chem. 193, 265-275. - 16. Schoenenderger, G. A. \& WACKER, W. E. C. (1968), in: Enzymes in Urine and Kidney (Dubach, U. C., Hrsg.) Bd. 2 „Aktuelle Probleme in der Klinischen Biochemie", S. 101-117, Verlag Hans Huber, Bern u. Stuttgart. - 17. Szaș, G. (1969), Enzymol. Biol. Clin. 10, 345-346. - 18. Szasz, G. (1969), Clin. Chem. 15, 124-135. 19. Klex, S. \& Klex, R. (1973), Deut. Med. Wochenschr. 36, 1646-1651. - 20. KLEY, R. \& BAHRE, G. (1973), IX. Symposium der Gesellschaft für Nephrologie, 19.-22. Sept., Basel.

\author{
Dr. R. Kley \\ Klinische Anstalten der RWTH Aachen \\ 51 Aachen
}

Goethestraße 27/29 